(eg renal function and weight), are important for safe prescribing.

These results have influenced the content of a regional guideline to enable healthcare professionals to make effective, evidence-based and patient-centred decisions about anticoagulation in patients with a life-limiting illness.

\section{WHAT METHODS ARE USED TO SCREEN AND IDENTIFY CACHEXIA AND IN WHICH HEALTHCARE SETTINGS?}

Ugochinyere Nwulu, Brian D Nicholson, Helene L Elliott-Button, Miriam J Johnson, Fliss EM Murtagh. University of Hull, University of Oxford

\subsection{6/spcare-2020-PCC.213}

Background Cachexia is a complex metabolic syndrome where unintentional weight loss is associated with chronic and malignant disease. Cachexia is associated with frailty, reduced quality of life and poor survival. Systematic identification of cachexia is lacking in practice though consensus definitions exist. The aim of the review is to describe the common tools and diagnostic criteria used to identify cachexia and to describe the healthcare settings in which screening and identification takes place.

Methods A systematic review of primary research studies published in English, 2008 to 2019, was conducted using MEDLINE, EMBASE, PsycINFO, and CINAHL.

Results Most studies were in cancer cachexia (84/98) and all were conducted in secondary care settings and five studies specifically in palliative care settings. Three studies assessed the risk of developing cachexia. All but one study used basic anthropometric measurements. Body composition was assessed in 68 studies where imaging techniques such as CT, MRI and BIA were used. Patients were also assessed for muscle strength, nutritional status, and inflammatory markers. Sixty-one studies used published diagnostic criteria and from 2011, under half of the cancer studies used the Fearon et al criteria (33/79 studies). In studies without a referenced criteria, a weight loss of $5 \%$ or more in the past 6 months was the most common inclusion criteria (13/37 studies).

Conclusions Cancer cachexia studies are increasingly adopting one consensus definition (Fearon et al) but not consistently. Few studies assessed cachexia risk, and none were conducted in primary care and few in palliative care settings. Most assessments were made in secondary care where active treatment for the associated disease is being undertaken. There is a need for detection of early markers of cachexia for those at risk coupled with the development of effective interventions for those with symptoms before the onset of refractory cachexia.

\section{QUALITY IMPROVEMENT PROJECT EVALUATING COMPLIANCE WITH LOCAL GUIDELINES IN PRESCRIBING BREAKTHROUGH DOSES OF STRONG OPIOIDS}

Rose O'Duffy, Apoorva Hallikeri. East Lancashire Teaching Hospitals

\subsection{6/spcare-2020-PCC.214}

Background Lancashire Medicines Management Group has clear regional guidance for prescribing strong opioids, adhering to generally accepted practice that the breakthrough dose of a strong opioid should be $1 / 6$ th to $1 / 10$ th of the regular prescription. We wished to assess if opioid prescribing in The Royal Blackburn Hospital (an Acute NHS Trust) was in line with these recommendations.

Methods The medication charts of all inpatients on five different acute medical wards at The Royal Blackburn hospital were reviewed. All strong opiate breakthrough prescriptions were identified and assessed for compliance with guidance. Following this, teaching sessions were provided for junior medical staff after which the data collection was repeated on the same wards.

Results In the first set of data, overall compliance with the guidelines was $63 \%$.

- $100 \%$ of the prescribing decision made by specialist teams i.e. the specialist palliative care nurses were correct.

- $50 \%$ of the prescribing decisions made by doctors were compliant.

In the second set of data overall compliance with the guidelines was $50 \%$.

- There was no input from specialist teams for any of the prescribing decisions.

- $50 \%$ of the decisions made by doctors were again complaint.

Conclusions These results demonstrate a failure of teaching on opioid prescription to materially alter the prescribing habits of junior medical staff. They also show the utility of specialist input, the absence of which led to a higher overall rate of sub-optimal prescribing decisions. Whilst we should continue to try to improve adherence to the guidelines by doctors, it remains imperative that clinical teams continue to consult with specialist teams i.e. palliative care.

\section{PRESCRIPTION AND USE OF ANTICIPATORY MEDICATIONS IN PATIENTS DYING AT HOME, IN HOSPICES AND IN HOSPITALS}

Karen Neoh, Moira Cookson, Adam Hurlow, Tammy Oxley, Amber Garnish. St Gemma's Hospice, Leeds Teaching Hospital Trust

\subsection{6/spcare-2020-PCC.215}

Background NICE guidance advises an 'individualised approach' to prescribing anticipatory medications including consideration of what medications are likely to be required depending on expected symptoms. We audited the prescription and administration of anticipatory medications (grouped into categories: benzodiazepines, opioids, anti-secretories and antiemetics) in patients ${ }^{6}$ last three days of life in three care settings.

Method A retrospective audit was undertaken and data collected for one month from Leeds Teaching Hospital Trust $(n=132)$, St Gemma's and Wheatfield's Hospices $(n=46)$ and Leeds Community Healthcare Trust $(n=31)$. Hospital data was extracted from e-medication prescriptions from adult inpatient deaths excluding sudden deaths. Hospice staff completed a proforma for all deaths. All community deaths undergo a mortality review where expected deaths were identified.

Results Prescribing practice varied across settings. 100\% of patients in hospices were prescribed all four medication categories. Prescription rates in hospital were lower and varied depending on drug group (benzodiazepine 49.2\%, opioid $57.6 \%$, anti-secretory $50 \%$ and anti-emetic 50.8\%). 